\title{
TURISMO CULTURAL, PATRIMONIO INMATERIAL Y ELEMENTOS QUE INCIDEN EN LA DIFERENTE SATISFACCIÓN DEL VISITANTE A PARTIR DEL ESTUDIO DEL SOMBRERO DE PAJA TOQUILLA
}

\author{
José Prada-Trigo* \\ Universidad de Concepción. Chile \\ Tomás López-Guzmán** \\ Universidad de Córdoba \\ Sandra Pesántez $z^{* * *}$ \\ Instituto Tecnológico Superior Luis Rogerio Gonzales. Ecuador \\ Jesús Pérez-Gálvez $z^{* * * *}$ \\ Universidad de Córdoba
}

\section{RESUMEN}

Pese a que existen varias aproximaciones a la relación entre patrimonio, características del turista y satisfacción, aún son necesarias interpretaciones más complejas, que indaguen en la comparación simultánea de variables de naturaleza diversa. En este sentido, el presente estudio busca dar una interpretación a las diferencias en cuanto a la valoración de los distintos ítems vinculados a la satisfacción de los turistas mediante la utilización de variables sociodemográficas, sociolaborales y de conocimiento previo sobre el destino, buscando identificar cuáles son más importantes. Estas cuestiones se aplican a un ámbito relativamente poco estudiado, como son las ciudades medias latinoamericanas que gozan de un reconocimiento como Ciudades Patrimonio Cultural de la Humanidad y, además, acogen un Patrimo-

Recibido: 2 de enero de 2017

Devuelto para su revisión: 27 de junio de 2017

Aceptado: 13 de octubre de 2017

Departamento de Geografía, Facultad de Arquitectura, Urbanismo y Geografía. Universidad de Concepción. Barrio Universitario s/n. CONCEPCIÓN (Chile).E-mail: jprada@udec.cl

** Área de Economía Aplicada, Facultad de Ciencias del Trabajo Universidad de Córdoba. C/ Adarve, 30 14071 CÓRDOBA (España).E-mail: tomas.lopez@uco.es

*** Instituto Tecnológico Superior Luis Rogerio Gonzales. C/ Luis Cordero 7-02. AZOGUES (Ecuador). E-mail: spesantezl@yahoo.com

***** Área de Economía Aplicada, Facultad de Derecho y Ciencias Económicas y Empresariales. Universidad de Córdoba. C/ Puerta Nueva, s/n. 14071 CóRDOBA (España).E-mail: dt1pegaj@uco.es 
nio Cultural Inmaterial también reconocido por la Unesco, elemento hacia el que se enfoca este trabajo. Para ello, se analizan e interpretan los resultados de una encuesta realizada en la ciudad de Cuenca (Ecuador) utilizando pruebas de normalidad. Los resultados ponen de manifiesto que el conocimiento previo del destino turístico y su condición de Patrimonio material o inmaterial son las cuestiones más significativas que influyen en la satisfacción del turista y el deseo de regresar.

Palabras clave: Patrimonio cultural inmaterial; características del turista; intención de retorno; Sombrero Paja Toquilla; Cuenca (Ecuador).

\title{
Cultural tourism, intangible patrimony and elements that affect the different satisfaction of the tourist from the study of the panama hat
}

\begin{abstract}
Although there are several approaches to study the relationship between heritage, characteristics of tourists and satisfaction, still more complex interpretations are needed, seeking simultaneously the comparison of variables of different nature. In this sense, this paper seeks to give an interpretation to the differences in the valuation of the different items related to tourist satisfaction through the use of sociodemographic, socio-labor and prior knowledge variables on the destination, seeking to identify which are more important. These issues apply to a relatively little studied area, such as the Latin American medium-sized cities that enjoy recognition as Cultural Heritage Cities and, in addition, host an Intangible Cultural Heritage also recognized by Unesco, an element to which it focuses this work. For this, the results of a survey carried out in the city of Cuenca (Ecuador) using normality tests are analyzed and interpreted. The results show that the previous knowledge of the tourist destination and its condition of material or immaterial heritage are the most significant issues that influence the satisfaction of the tourist and the desire to return.
\end{abstract}

Keywords: Intangible cultural heritage; tourist profile; intention to return; Panamá hat; Cuenca (Ecuador).

\section{INTRODUCCIÓN}

El incremento del turismo y de la importancia dentro del mismo de los destinos patrimoniales y culturales es un elemento que tiene un fuerte peso en la mejora de las condiciones de vida de muchos territorios, especialmente en áreas en desarrollo, donde puede suponer un incentivo a la redistribución de la riqueza y el crecimiento de la producción local (Telfer y Sharpley, 2008). Sin embargo, junto con el incremento de la atracción y la acogida de turistas, resulta necesario también mejorar su grado de satisfacción con la visita, elemento que tiene una influencia fundamental en su intención de regreso y en la recomendación del destino a otros visitantes.

Algunos trabajos han analizado la distinta satisfacción que indican los turistas, vinculándola con características como el sexo, el origen o el nivel de ingresos de los visitantes (Wearing y Wearing, 1996; McCleary, Weaver y Hsu, 2006). Sin embargo, debido a la utilización de enfoques demasiado concretos, existen límites en algunos de estos trabajos, 
por lo que resulta necesario llevar a cabo estudios más amplios que añadan otros factores explicativos (sociales, de conocimiento previo sobre el destino...) que aún no se han trabajado o no se han relacionado con esas otras características. En esta línea, el presente estudio busca dar una interpretación a las diferencias en cuanto a la valoración de los distintos ítems vinculados a la satisfacción de los turistas mediante la utilización de variables sociodemográficas, sociolaborales y de conocimiento previo sobre el destino, buscando identificar cuáles son más importantes. Además, el trabajo aplica estas cuestiones a un ámbito relativamente poco estudiado, como son las ciudades medias latinoamericanas que gozan de un reconocimiento como Ciudades Patrimonio Cultural de la Humanidad y, además, acogen un Patrimonio Cultural Inmaterial también reconocido por la Unesco, elemento hacia el que se enfoca este trabajo. De esta forma, los objetivos de esta investigación son: [i] identificar el peso de los factores vinculados con las características sociodemográficas, sociolaborales o cuestiones relacionadas con el conocimiento previo del destino a la hora de valorar la experiencia turística; y [ii] identificar la relación de elementos concretos, discernibles a partir de los factores mencionados, en la valoración de la visita turística a Cuenca, comparando los resultados con trabajos previos.

Para ello, se aplica una metodología basada en la encuesta estructurada a los turistas nacionales y extranjeros que se encontraban visitando espacios relacionados con el sombrero de paja toquilla, declarado por la Unesco como Patrimonio Cultural Inmaterial de la Humanidad en 2012. A partir de ello, mediante análisis con pruebas no paramétricas, se trata de identificar cuáles son los factores (edad, sexo, nivel de instrucción, nivel de ingresos, conocimiento previo de la ciudad, conocimiento previo del sombrero paja toquilla, etc.) agrupados en tres categorías principales, que explicarían la diferente valoración que se da a los elementos vinculados a la satisfacción con la visita turística (precios, atención, hoteles, museos...).

De esta forma, a continuación, se lleva a cabo una revisión de la literatura que pone de relieve los alcances y limitaciones de los análisis de la satisfacción mediante la utilización de factores sociales o económicos para medir las diferencias. Después se presenta el caso de estudio de la ciudad de Cuenca (Ecuador), lugar vinculado tanto al patrimonio cultural material como al inmaterial a través del mencionado sombrero de paja toquilla, que se relaciona fuertemente con este territorio. Finalmente, se lleva a cabo un análisis e interpretación de los resultados, que pone de relieve el diferente peso de estos factores para explicar las distintas valoraciones de los ítems analizados, terminando el trabajo con una discusión de los resultados y unas conclusiones.

\section{REVISIÓN DE LA LITERATURA}

Un destino turístico puede definirse como una amalgama de productos y servicios turísticos consumidos bajo una marca y que ofrece a los visitantes una experiencia integral, que se valora subjetivamente en base a su itinerario, trasfondo cultural, propósito de la visita, experiencias del pasado, etc. (Zabkar, Makovec y Dmitrovic, 2010). En este sentido, la satisfacción se define como una evaluación posterior a la compra realizada por los consumidores en cuanto a su experiencia con el producto, y surge de la comparación de éste con las expectativas previas (Chen y Chen, 2010). De hecho, proveer 
servicios de alta calidad y asegurar la satisfacción del consumidor se considera como un factor fundamental para el éxito de los destinos turísticos (Kee, Wan y Ho, 2007).

De esta forma, la variable clave de la lealtad hacia un destino turístico es la satisfacción del turista, tanto como elemento que dirige directamente la intención de regresar, como en las recomendaciones a terceras personas, o en su papel de variable modeladora de la relación entre la imagen que se tiene de un destino y su realidad. Así, una experiencia positiva en cuanto a servicio, productos turísticos, viaje y otros recursos que un destino turístico puede proporcionar, llevaría a una mayor intención del turista en repetir la visita, además de generar una promoción positiva del destino (Sanz-Ibáñez y Clavé, 2014). En este sentido, la satisfacción y la intención de volver a un destino están en parte determinados por la valoración de las características del destino (Alegre y Garau, 2010), siendo elementos básicos para evaluar el desempeño de un lugar o destino turístico (Yoon y Uysal, 2005). Por esto, los factores que podrían explicar la diferente satisfacción de los turistas con un lugar constituyen uno de los elementos que más interés ha suscitado entre los académicos, especialmente en un contexto en el que el mercado turístico se ha vuelto crecientemente competitivo, lo que lleva a plantear la necesidad de fomentar la fidelización de los visitantes (Chen y Chen, 2010; Alegre y Cladera, 2009) o la búsqueda de elementos sobre los que enfocar la publicidad en torno a los atractivos de un lugar.

La mayoría de estudios en la actualidad proponen que la satisfacción del cliente y el apego a un destino específico derivan de la experiencia vivida, pudiendo verse afectados por su punto de vista personal, su sentimentalismo y su expectativa propia (Yuksel, Yuksel y Bilim, 2010). De hecho, la experiencia de los turistas se ha convertido en un concepto clave dentro del turismo cultural y patrimonial, donde la satisfacción se encuentra determinada a menudo por la experiencia global obtenida, lo que incluye entretenimiento, cultura, educación e interacciones sociales (De Rojas y Camarero, 2008). Por ello, desde determinadas perspectivas (Schofield y Thompson, 2007) es necesario determinar los factores que afectan a la motivación y experiencias del visitante.

En este sentido, cuestiones como el género del turista y su influencia en la satisfacción se han subrayado en algunos estudios (Breathnach et al., 1994; Wearing y Wearing, 1996) pese a que los resultados señalan que este elemento puede haberse sobrevalorado (Schofield y Thompson, 2007). En este sentido, Huh, Uysal y McCleary (2006) llevaron a cabo un análisis de la satisfacción o insatisfacción del destino patrimonial cultural en función del perfil del turista con variables que se correlacionan, como la edad y el sexo, hallando evidencias de interés. De igual manera, Formica y Uysal (1998) encontraron también diferencias significativas entre turistas en estos aspectos. Algunos otros estudios también han puesto de relieve la importancia de la edad como variable determinante, bien para la valoración de la experiencia (Kim y Morrison, 2005), bien para determinar su influencia en el gasto (Leeworthy et al., 2001). Mientras que los factores más comunes para realizar estos análisis son los ingresos, el nivel de educación y el sexo, los estudios que han utilizado factores como el área de origen son más escasos (McCleary, Weaver y Hsu, 2006), a pesar de constituir un análisis bastante válido y relativamente sencillo de realizar (Kee, Wan y Ho, 2007). Pese a esto, el origen cuenta también con algunos trabajos que manifiestan su influencia sobre el comportamiento (Formica y Uysal, 1996; Lee et al., 2004). 
De esta forma, en los estudios que han teorizado sobre si existe o no influencia de los valores culturales en el comportamiento del turista las hipótesis han sido validadas y, además, se ha podido plantear que las variables culturales agrupan diferentes ámbitos, tales como la cultura global, la nacional y la local o regional (Weiermair, 2000). Este tipo de distancia cultural es importante ya que los turistas que viajan más de una vez al mismo lugar deberían ser menos sensitivos a estas "diferencias culturales" (Oom et al., 2011) y a su vez impulsan al sector de destino a desarrollar una nueva forma de encarar sus visitas y superar sus diferencias. Este último elemento determinaría en cierto modo la percepción de la calidad del servicio y su valoración o satisfacción con el mismo (Kerstetter et al., 2001; Turner et al., 2002).

En un caso u otro, parece hoy evidente que se está generando una creciente "segmentación" de las categorías de turistas, lo que conduciría a establecer distintas valoraciones de los destinos (Huh, Uysal y McCleary, 2006), contribuyéndose de esta forma a obtener una mejor visión de los mismos, que permita comprender fenómenos como la repetición de la visita. Existe una gama de factores culturales, de clase social, socialización y personalidad que influyen en el nivel de conocimiento, disposición e interés del turista. De este modo, surge la necesidad de investigar las relaciones existentes entre las motivaciones culturales y el comportamiento y valoración de este tipo de turistas, reconociendo varios estudios que muchos turistas están más altamente motivados por los destinos culturales que otros (Cordente et al. 2011; McKercher y du Cros, 2003). Por lo tanto, el éxito de un destino turístico se encuentra en la satisfacción del turista, que no solo debe ser medida por la elección del mismo sino por la decisión de volver (Kozak y Rimmington, 2000), para lo cual las cuestiones vinculadas a las características sociales, económicas o demográficas de los visitantes pueden jugar un papel importante.

En relación con el patrimonio cultural inmaterial, la Organización Mundial del Turismo señala que es necesario llevar a cabo estudios desde la academia que permitan un seguimiento de los efectos del turismo cultural, haciendo acopio y analizando datos sobre los productos asociados al Patrimonio Inmaterial de la Humanidad (UNWTO, 2012). En cuanto a los estudios empíricos centrados en la relación entre el patrimonio y el turismo, la literatura científica se ha centrado básicamente en el patrimonio material y en las ciudades Patrimonio de la Humanidad (en adelante WHS por sus siglas en inglés World Heritage Site). Así, por ejemplo, y en relación con el WHS, pueden encontrarse estudios en ámbitos tan variados como Ecuador (López-Guzmán, et al., 2017), Serbia (Krasojevic y Djordjevic, 2017) o Vietnam (Nguyen y Cheung, 2014). Sin embargo, y en relación con el Patrimonio Inmaterial de la Humanidad, no son muchos los estudios académicos realizados debido en parte a la dificultad de relacionar este tipo de patrimonio a un territorio concreto (Vidal González, 2008).

Pese a esto, puede destacarse la investigación de Gómez Schettini, Almirón y González Bracco (2011) que analiza el tango como recurso turístico en la ciudad de Buenos Aires. A su vez, Bille (2012) analiza la relación de los beduinos de Petra y el Wadi Rum en Jordania como Patrimonio cultural inmaterial y su relación tanto con la potenciación con el turismo como su sinergia con la declaración de la ciudad de Petra como WHS. Por su parte, dentro del contexto español, González Santa-Cruz y López-Guzmán (2017) analizan la relación entre el turismo y la Fiesta de los Patios en la ciudad de Córdoba. Finalmente, algunos trabajos recientes se centran más bien en las estrategias para transformar activos de este Patrimonio inmaterial en productos culturales, destacando el trabajo de Villacis-Mejia, et 
al. (2017) plantean la necesita de diseñar productos turísticos exclusivamente centrados en el patrimonio inmaterial. Sin embargo, como se dijo, existe una ausencia de trabajos sobre los turistas en el contexto del patrimonio cultural inmaterial en relación al ámbito latinoamericano y a los distintos factores que pueden motivar su distinta valoración.

\section{CASO DE ESTUDIO}

Santa Ana de los Cuatro Ríos de Cuenca, la tercera ciudad más importante del Ecuador, con cerca de medio millón de habitantes, se encuentra ubicada en la cordillera Andina a 2.550 metros sobre el nivel del mar (figura 1) enclavada en un valle, y rodeada de una estructura montañosa que alberga un extenso sistema lacustre que baña a la ciudad a través de sus cuatro ríos, de donde proviene su nombre. Este lugar se ha constituido en un referente cultural y político del país desde la época pre hispánica con el asentamiento del pueblo cañarí, a quien se sobrevino la expansión inca y la posterior conquista española (Vanegas, 2008,) a partir de la cual fue bautizada con su nombre actual.

$\mathrm{Su}$ diversidad cultural ha sido un elemento de indiscutible valor y uno de los atractivos de esta ciudad, lo que ha propiciado un amplio desarrollo de las artes y las ciencias que se evidencia en sus ruinas arqueológicas, sus museos de arte religioso y sus iglesias de los periodos colonial y republicano, cuyas fachadas e interiores son claras representaciones del arte barroco y renacentista. De esta forma, en 1999 la ciudad fue declarada Patrimonio Cultural de la Humanidad por la Unesco, considerándose para ello la implantación en Cuenca de los principios de la planificación urbana del renacimiento en América, la fusión alcanzada por diferentes sociedades y culturas de América Latina, que se manifiesta de manera notable en la traza y el paisaje urbano de Cuenca, y el hecho de que Cuenca es un ejemplo excepcional de ciudad colonial española, planificada y situada en zonas de interior. Esto hizo de Cuenca la segunda ciudad Patrimonio Cultural de la Humanidad en Ecuador después de Quito (Unesco, 2015).

Además de por su patrimonio construido, la ciudad destaca por sus eventos culturales, como la Bienal Internacional de Pintura, las actuaciones de la Orquesta Sinfónica, sus grupos de ballet y folklore local y nacional; el Festival Internacional de Cine de La Orquídea o los Festivales Internacionales de Danza del Austro, desde 2010, y el Festival Internacional y de Artes Escénicas, desde 2007. De igual manera sucede con sus artesanías, en donde productos de alfarería, orfebrería, o tejidos de distintos materiales como el mimbre son muy apreciados por los habitantes de la zona y los turistas. Sin embargo, entre todos estos destaca el sombrero de paja toquilla, que recibió la declaratoria de Patrimonio Cultural Inmaterial de la Humanidad por la Unesco en 2012 (Unesco, 2015).

El sombrero de paja toquilla, conocido como Panama Hat, fue adoptado por la tradición productiva de los indígenas de las provincias del Azuay y Cañar principalmente, a partir de 1835, aunque su elaboración en Ecuador se remonta siglos atrás (Mintur, 2014). Su fino tejido y delicado acabado pronto lo catalogaron como un elemento apetecido y de gran renombre a nivel internacional, convirtiéndose en un producto de exportación para este país y ocupando el primer lugar de los productos exportados por el Ecuador en 1944 (Mintur, 2014). Su elaboración sigue siendo parte esencial de la cultura cuencana y se ha convertido en un importante ingreso económico para sus pobladores y los de varios cantones de 
la provincia del Azuay, principalmente del cantón Sigsig (a 60 km de Cuenca) en donde el sombrero tiene gran relevancia, siendo elaborado en su mayoría por mujeres. El cantón Sigsig ha estado íntimamente relacionado al tejido de sombreros de paja toquilla desde el siglo XIX y actualmente representa una importante riqueza turística y cultural muy vinculada a Cuenca (Aguilar, 2011). El proceso de fabricación del sombrero de paja toquilla así como su variedad de modelos, extensa gama de colores y usos, atraen diariamente a turistas nacionales y extranjeros dispuestos a adquirirlos y conocer sus orígenes, así como a sus maestros artesanos, lo que ha permitido un desarrollo económico y social de las zonas en torno al turismo cultural inmaterial. Mientras que en el Sigsig se conserva la tradición del tejido de la paja toquilla en la ciudad de Cuenca se concentran las empresas que realizan los acabados a los sombreros, exportan los sombreros y cuentan con museos y tiendas enfocados a este producto. También existen un museo del municipio de Cuenca y algunos espacios dedicados al estudio este patrimonio y de otros saberes, como el Centro Interamericano de Artes Populares, junto con unas mejoras comunicaciones, por lo que la atracción de turistas es mayor.

\section{Figura 1 \\ SITUACIÓN DE LA CIUDAD DE CUENCA}

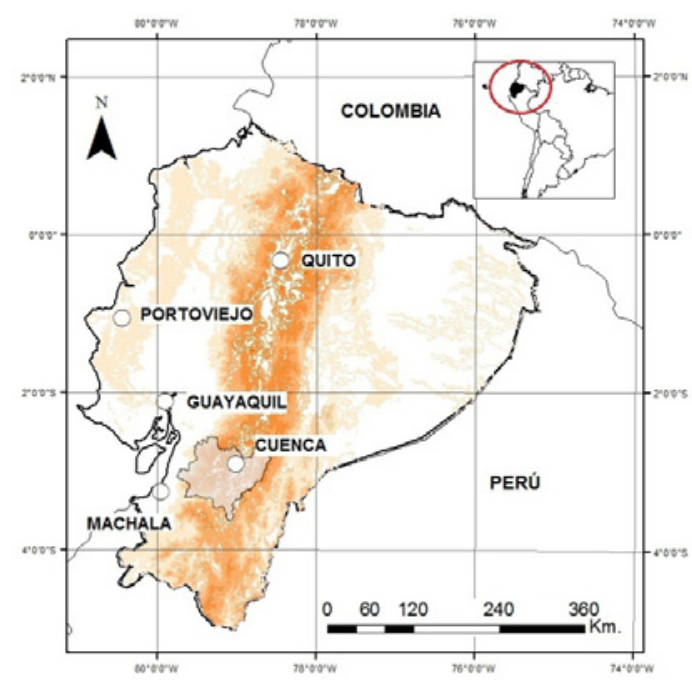

Elaboración propia.

La importancia de Cuenca como destino dentro del Ecuador se debe no solamente a sus atractivos patrimoniales, sino también a la existencia de una importante planta hotelera y de restauración, compuesta por más de 150 hoteles y de 400 restaurantes dispersos por toda la ciudad pero con una presencia muy evidente en el centro de la ciudad (Prada-Trigo et al., 2015), que atraen hacia Cuenca a más de 200.000 turistas cada año, calculándose en cerca de 4.400 los empleos que genera en la ciudad el sector turístico (López-Guzmán, et al., 2017). Estos autores indican el predominio de un turista caracterizado por ser menor de 40 años, asalariado o profesional liberal y con un alto nivel de formación. De esta forma, Cuenca se ha 
consolidado como un destino de cierto prestigio, ligado al patrimonio, la cultura y lo culinario, generando un flujo de visitantes que valora estas tradiciones, a menudo vinculadas a otros elementos exóticos intangibles como el consumo del cuy o conejillo de indias (Prada-Trigo, 2017).

\section{METODOLOGÍA}

De manera creciente, como se dijo antes, existe un interés en conocer las características, tipologías, valoración y motivaciones de los turistas que visitan destinos culturales, al no ser este un grupo homogéneo sino un compuesto variado de viajeros que arriban a un espacio por diferentes motivaciones y con perfiles heterogéneos. En este sentido, entre las ventajas de levantar información estadística se encuentra el conseguir una mejor comprensión de sus gustos y necesidades para adaptar así la oferta del destino a estos perfiles y requerimientos (Pulido-Fernández y Sánchez-Rivero, 2010). En este artículo se presentan los resultados de una investigación llevada a cabo en la ciudad de Cuenca, Ecuador. A nivel metodológico, se ha desarrollado un trabajo empírico con encuestas personales estructuradas, dirigidas a los turistas que se encontraban visitando Cuenca en diferentes puntos turísticos relacionados con el sombrero de paja toquilla (museos, tiendas, fábricas, etc.). El cuestionario en papel ha sido la herramienta utilizada para la recogida de información, mediante preguntas en una escala tipo Likert de 1 a 5. Este cuestionario, previamente a su implementación, pasó por un muestreo piloto y una mejora después de analizar los resultados. La población objetivo para este trabajo se ha identificado como los turistas ecuatorianos o extranjeros que se encontraban en la ciudad de Cuenca, visitando lugares vinculados al sombrero de paja toquilla, durante los meses de octubre y noviembre de 2015. Como técnica de muestreo, se ha utilizado el muestreo no probabilístico y de conveniencia. Los interceptados han sido seleccionados tratando de abarcar distintas capas sociodemográficas y un perfil lo más amplio posible, pese a que el hecho de realizar las encuestas mayoritariamente entre semana, ha supuesto un sesgo en favor de los turistas extranjeros. En la tabla 1 aparecen los detalles de los aspectos técnicos de la investigación, cuyos resultados se analizan a continuación.

\section{Tabla 1}

\section{DISEÑO DE LA MUESTRA DE LA INVESTIGACIÓN}

\begin{tabular}{|l|l|}
\hline Universo & Turistas y excursionistas de la ciudad de Cuenca \\
\hline Ámbito & Municipal, Cuenca (Ecuador) \\
\hline Método de recogida de información & Encuesta personal con cuestionario estructurado \\
\hline Unidad muestral & Turistas y excursionistas de la ciudad de Cuenca \\
\hline Tamaño de la población & No definido \\
\hline Tamaño de la muestra & 850 \\
\hline Tipo de muestreo & No probabilístico de conveniencia \\
\hline Nivel de Confianza & $95 \%$ \\
\hline Error muestral & $+/-3,4 \%$ \\
\hline Trabajo de campo & Meses de octubre y noviembre de 2015 \\
\hline Número de encuestas válidas realizadas & 858 \\
\hline
\end{tabular}

Elaboración propia. 


\section{ANÁLISIS DE LOS RESULTADOS}

Como se indicaba, el objetivo de este artículo es conocer la relación entre la diferente valoración de varios ítems relacionados con la satisfacción respecto al viaje por un lado, y las características sociodemográficas, sociolaborales o de conocimiento previo del destino y su patrimonio cultural por otro lado. Para esto, primeramente se compararon las valoraciones medias y la desviación típica de cada uno de los diecinueve ítems analizados (tabla 2), observándose que los aspectos que muestran una mayor valoración son la satisfacción con el viaje (4,39 sobre 5) y la opinión sobre la ciudad de Cuenca como un destino patrimonial $(4,48)$. Además, otros elementos como la hospitalidad y el trato de la población local, la limpieza de la ciudad, los hoteles y restaurantes, la seguridad y la conservación del entorno también tuvieron puntuaciones superiores a cuatro. Por el contrario, varios aspectos relacionados con el sombrero de paja toquilla, como el cantón Sigsig $(2,61$ sobre 5) o las fábricas y museos relacionados con el sombrero de paja toquilla $(3,28$ y 3,22 respectivamente) obtuvieron las menores valoraciones, junto a otras cuestiones como el precio del viaje $(3,55)$ o la señalización de los lugares turísticos $(3,59)$.

A continuación, se recurrió a la prueba de normalidad de Kolmogorov-Smirnov (tabla 2) con la intención de contrastar la hipótesis de normalidad de la distribución en el caso de las respuestas vinculadas a la satisfacción. En este sentido, al arrojar los resultados una significatividad $<0,005$ la muestra no refleja una distribución normal en ningún caso, por lo que se aplicaría la prueba de Kruskall-Wallis, válida para comparar datos con más de dos variables en relación a ese ítem y la prueba de Mann-Whitney para elementos donde solamente existan dos variables (como es el caso de "sexo" o de "existencia de un conocimiento previo sobre el destino") para el análisis de las diferencias en las respuestas entre grupos base a las características de los turistas.

Tabla 2

\section{PROMEDIO, DESVIACIÓN TÍPICA Y PRUEBAS DE NORMALIDAD DE KOLMOGOROV-SMIRNOV PARA LAS VARIABLES RELACIONADAS CON LA SATISFACCIÓN DE LOS TURISTAS}

\begin{tabular}{|l|c|c|c|c|c|}
\hline & $\mathrm{N}$ & Media & $\begin{array}{c}\text { Desv. } \\
\text { típ. }\end{array}$ & $\begin{array}{c}\text { Z de } \\
\text { Kolmogorov- } \\
\text { Smirnov }\end{array}$ & $\begin{array}{c}\text { Sig. } \\
\text { asintót. } \\
\text { (bilateral) }\end{array}$ \\
\hline Cantón de Sigsig y la elaboración del sombrero & 765 & 2,61 & 1,344 & 4,776 & 0,000 \\
\hline $\begin{array}{l}\text { Fábricas de sombreros de paja toquilla de } \\
\text { Cuenca }\end{array}$ & 791 & 3,22 & 1,219 & 5,607 & 0,000 \\
\hline $\begin{array}{l}\text { Museos relacionados con el sombrero de paja } \\
\text { toquilla }\end{array}$ & 795 & 3,28 & 1,206 & 5,015 & 0,000 \\
\hline Precio del viaje & 822 & 3,55 &, 989 & 5,660 & 0,000 \\
\hline Señalización & 817 & 3,59 & 1,092 & 5,684 & 0,000 \\
\hline Conexión a Internet & 825 & 3,68 & 1,122 & 6,004 & 0,000 \\
\hline Puntos de información y atención al visitante & 822 & 3,70 & 1,056 & 6,527 & 0,000 \\
\hline Servicio de Taxis & 819 & 3,71 & 1,099 & 6,358 & 0,000 \\
\hline
\end{tabular}




\begin{tabular}{|l|c|c|c|c|c|}
\hline Actividades culturales y espectáculos & 810 & 3,73 & 1,095 & 6,020 & 0,000 \\
\hline Zona comercial de productos artesanos & 814 & 3,87 & 1,021 & 6,489 & 0,000 \\
\hline Satisfacción fabricación sombrero paja toquilla & 794 & 3,91 & 1,050 & 6,568 & 0,000 \\
\hline Seguridad ciudadana & 835 & 4,06 &, 915 & 6,922 & 0,000 \\
\hline Conservación del entorno & 818 & 4,09 &, 905 & 7,078 & 0,000 \\
\hline Alojamiento & 823 & 4,11 &, 900 & 6,822 & 0,000 \\
\hline Restaurantes & 829 & 4,11 &, 873 & 6,741 & 0,000 \\
\hline Limpieza & 833 & 4,15 &, 893 & 7,039 & 0,000 \\
\hline Hospitalidad/Trato & 832 & 4,23 &, 864 & 7,690 & 0,000 \\
\hline Satisfacción general viaje & 832 & 4,39 &, 836 & 8,876 & 0,000 \\
\hline Opinión Cuenca & 778 & 4,48 &, 751 & 9,899 & 0,000 \\
\hline
\end{tabular}

Elaboración propia.

A partir de ello, se ha llevado a cabo un análisis de estos elementos en base a tres diferentes tipologías de rasgos que permiten clasificar a los turistas. En primer lugar, se han utilizado rasgos de tipo sociodemográfico, donde el lugar de origen (agrupado en cuatro grandes zonas: Ecuador, América Latina, Europa y América del Norte), la edad (dividida en 5 categorías) y el sexo se han utilizado como indicadores para diferenciar la importancia de la respuesta de los turistas. En segundo lugar, se han aplicado elementos de tipo sociolaboral, como son el nivel de estudios (3 categorías), la categoría profesional del turista (7 divisiones) y su nivel de renta (5 categorías). En tercer lugar, se ha generado un filtro con rasgos vinculados al conocimiento previo del destino, a partir de que se hubieran dado visitas previas a la ciudad de Cuenca (3 categorías en función de las veces que se había visitado Cuenca), de que se conociese previamente al viaje la categoría de Ciudad Patrimonio de la Humanidad de Cuenca o de que se tuviera información, previo al viaje, de que el sombrero de paja toquilla era un producto vinculado al territorio de Cuenca y su entorno (respuestas tipo sí/no).

Para esto, se decidió aplicar la prueba de Kruskal-Wallis, un método no paramétrico para probar si un grupo de datos proviene de la misma población o refleja diferencias significativas en función de algún parámetro determinado. Este método, que se utiliza cuando la distribución de la muestra no es homogénea, es idéntico al ANOVA y constituye una extensión de la prueba de la U de Mann-Whitney para tres o más grupos. En este sentido, a continuación se aplica la U de Mann-Whitney para las variables que sólo permiten generar dos respuestas (sexo y conocimiento previo o no del sombrero de paja toquilla como patrimonio cultural inmaterial y como elemento vinculado a la ciudad de Cuenca) y la prueba de Kruskal-Wallis para el resto. Este análisis estadístico busca, por lo tanto, los factores o motivos que pueden establecer las distintas valoraciones de los ítems analizados, vinculados a la satisfacción con el destino (tabla 3).

Los resultados evidencian que los llamados rasgos vinculados al conocimiento serían los que se relacionarían con un mayor número de diferencias en la valoración de los ítems analizados, en especial en el caso de la existencia de conocimiento del sombrero de paja toquilla como un producto vinculado a la identidad cuencana, que explicaría distintas valoraciones en nueve aspectos, cuatro de los cuales se relacionan directamente con el sombrero de paja toquilla: museos, fábricas, valoración del cantón Sigsig y del sombrero 
de paja toquilla; junto con otras cuestiones como la limpieza de Cuenca, el servicio de taxis, las actividades culturales y espectáculos, los productos artesanos comercializables o la conexión a internet. Por su parte, la existencia de visitas previas se relacionaría con seis valoraciones diferentes, en general más conectadas con cuestiones prácticas como el precio del viaje, la señalización de los elementos turísticos, el servicio de taxis, las actividades culturales y espectáculos o los productos artesanos comercializables, junto al cantón Sigsig. Finalmente, el conocimiento previo de Cuenca como una ciudad Patrimonio Cultural de la Humanidad se vincularía con la existencia de diferencias en tres elementos relacionados con el sombrero paja toquilla como son los museos, las fábricas y el cantón Sigsig, junto con otros dos: el propio viaje a Cuenca, lo que coincide con la existencia de un conocimiento previo de la ciudad como destino patrimonial, y el servicio de taxis.

Seguido de esto, a cierta distancia destacaría el peso de los rasgos sociodemográficos, especialmente del origen, como factores explicativos de las diferentes valoraciones. En el caso de éste, marcaría ocho diferencias en relación con la valoración: el precio del viaje, la satisfacción con la conservación del entorno, la señalización de los elementos turísticos, la limpieza, las actividades culturales, el servicio de taxis y la zona comercial. Estos, en general, son aspectos muy subjetivos en tanto el origen puede dar pie a diferencias en cuanto a la consideración de un servicio o producto como caro, a los estándares de limpieza existentes o a la consideración sobre la buena o mala conservación del entorno. Por ejemplo, para una persona que proviene de otra ciudad ecuatoriana, la percepción habitual de Cuenca es la de una ciudad bastante limpia, segura y ordenada, mientras que esta consideración puede ser muy distinta para turistas provenientes de otros países o contextos donde dichos elementos son más tenidos en cuenta.

Finalmente, puede considerarse que otros rasgos sociodemográficos como la edad y el sexo (cada uno explicando tres valoraciones diferentes) y los rasgos sociolaborales apenas tuvieron importancia en la interpretación de las diferentes valoraciones. En este sentido, la renta influyó solamente en la valoración del precio del viaje, de las zonas comerciales y de la señalización y puntos de información (lo que parece lógico en los dos primeros casos), mientras que el nivel de estudios y la categoría profesional no tuvieron apenas relevancia. Además de esto, existen tres ítems para los que no se ha encontrado ningún factor determinante de las diferencias: la calificación del alojamiento, de la restauración y la satisfacción con la ciudad de Cuenca como Ciudad Patrimonio de la Humanidad. Las causas de ello podrían ser dos diferentes: por un lado, el hecho de que éstas sean cuestiones tan interiorizadas (por ser el alojamiento y la restauración las necesidades más básicas de un desplazamiento turístico) que se hayan convertido en homogéneas independientemente cuestiones sociodemográficas, sociolaborales o vinculadas al conocimiento del destino. Por otro lado, existe la posibilidad de que haya otras variables no identificadas en la encuesta que expliquen estos elementos y puedan establecer distintas valoraciones de los mismos. En el caso de la satisfacción con Cuenca como Ciudad Patrimonio Cultural de la Humanidad, el hecho de que este ítem presente la valoración más alta de todos (4,48 sobre 5) supone que exista una importante concordancia en la calificación que se le dio al mismo, siendo un elemento que genera satisfacción en general en casi todos los turistas, y no siendo sencillo discernir si alguna característica de los visitantes puede influir en una mejor o peor valoración, al existir una aquiescencia general en torno a la satisfacción con este destino. 


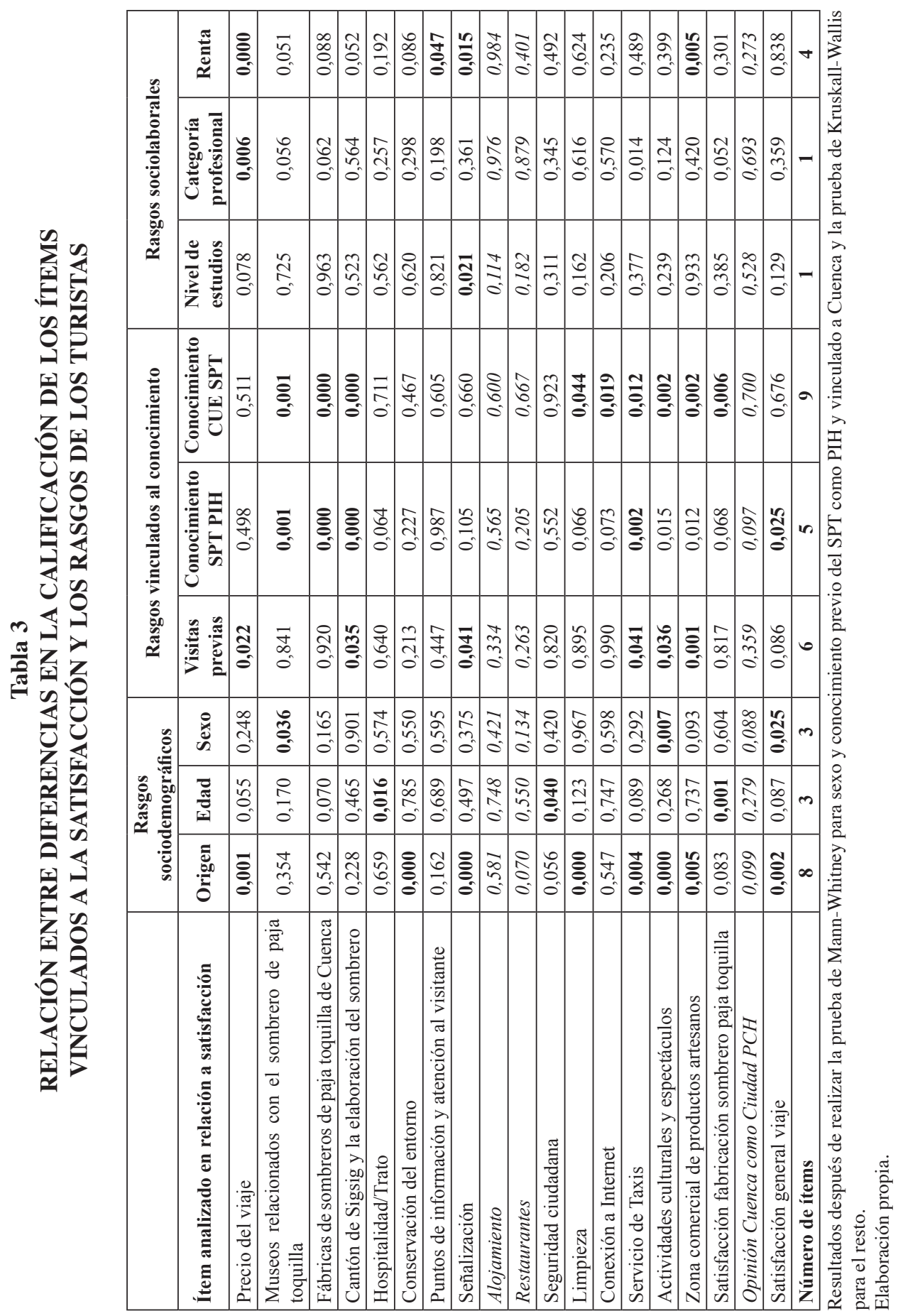


Por último, se aislaron los cuatro factores que parecían generar mayor número de diferencias en la valoración de los ítems analizados para comparar las calificaciones medias de cada subgrupo: origen, número de visitas a la ciudad, conocimiento previo del sombrero de paja toquilla como Patrimonio Inmaterial de la Humanidad y conocimiento previo del sombrero de paja toquilla como producto vinculado a Cuenca. En primer lugar, como puede observarse en la tabla 4, existen diferencias significativas en los ocho ítems que presentan valoraciones desiguales, marcadas en negrita.

\section{Tabla 4}

\section{VALORACIÓN MEDIA DE LOS ELEMENTOS VINCULADOS A LA SATISFACCIÓN SEGÚN ORIGEN DEL TURISTA}

\begin{tabular}{|l|c|c|c|c|}
\hline Ótigen del turista & Ecuador & $\begin{array}{c}\text { América Central } \\
\text { y Latina }\end{array}$ & Norteamérica & Europa \\
\hline Precio del viaje & 3,38 & 3,54 & 3,72 & 3,54 \\
\hline $\begin{array}{l}\text { Museos relacionados con el sombrero de } \\
\text { paja toquilla }\end{array}$ & 3,34 & 3,15 & 3,27 & 3,39 \\
\hline $\begin{array}{l}\text { Fábricas de sombreros de paja toquilla } \\
\text { de Cuenca }\end{array}$ & 3,28 & 3,18 & 3,16 & 3,32 \\
\hline $\begin{array}{l}\text { Cantón de Sigsig y la elaboración del } \\
\text { sombrero }\end{array}$ & 2,69 & 2,52 & 2,66 & 2,46 \\
\hline Hospitalidad/Trato & 4,22 & 4,27 & 4,29 & 4,23 \\
\hline Conservación del entorno & 4,23 & 4,27 & 3,92 & 3,94 \\
\hline $\begin{array}{l}\text { Puntos de información y atención al } \\
\text { visitante }\end{array}$ & 3,79 & 3,63 & 3,69 & 3,59 \\
\hline Señalización & 3,88 & 3,57 & 3,46 & 3,34 \\
\hline Alojamiento & 4,08 & 4,12 & 4,08 & 4,15 \\
\hline Restaurantes & 4,15 & 4,10 & 4,18 & 4,02 \\
\hline Seguridad ciudadana & 4,13 & 4,16 & 3,97 & 4,04 \\
\hline Limpieza & 4,30 & 4,25 & 4,02 & 4,10 \\
\hline Conexión a Internet & 3,72 & 3,66 & 3,76 & 3,56 \\
\hline Servicio de Taxis & 3,77 & 3,53 & 3,86 & 3,62 \\
\hline Actividades culturales y espectáculos & 3,77 & 3,45 & 4,06 & 3,45 \\
\hline $\begin{array}{l}\text { Zona comercial de productos } \\
\text { artesanos }\end{array}$ & 4,00 & 3,80 & 3,97 & 3,72 \\
\hline $\begin{array}{l}\text { Satisfacción fabricación sombrero paja } \\
\text { toquilla }\end{array}$ & 3,88 & 3,80 & 4,03 & 3,88 \\
\hline Opinión Cuenca como Ciudad PCH & 4,55 & 4,51 & 4,52 & 4,36 \\
\hline Satisfacción general viaje & 4,51 & 4,31 & 4,48 & 4,28 \\
\hline $\begin{array}{l}\text { PROMEDIO VALORES QUE } \\
\text { ARROJAN DIFERENCIAS }\end{array}$ & $\mathbf{3 , 9 8}$ & $\mathbf{3 , 8 4}$ & $\mathbf{3 , 9 4}$ & $\mathbf{3 , 7 5}$ \\
\hline
\end{tabular}

Elaboración propia. 
En este sentido, el precio del viaje les pareció mucho más caro a los ecuatorianos que al resto de turistas, siendo la mejor valoración la de los turistas norteamericanos. Esto puede ser debido al hecho de que Cuenca se considera dentro del país como una ciudad cara en relación al resto, incluidas Quito y Guayaquil, por lo que la percepción del turista nacional puede diferir significativamente de la del extranjero. Algo similar, pero en sentido contrario, sucede con la valoración de la limpieza, de la conservación del entorno, de la señalización de la ciudad y de los productos artesanales. Como se dijo previamente, Cuenca tiene fama dentro del Ecuador por ser una ciudad limpia, ordenada y bien conservada, lo que comparativamente da una valoración muy buena a estos ítems por parte de los turistas nacionales. Por el contrario, en otros países con un mayor desarrollo de cuestiones como la separación de residuos, una señalización más completa de las rutas turísticas, un mayor respeto al patrimonio edificado, etc. esta valoración resultaría más negativa, como puede observarse en los resultados. En el caso de los productos artesanales, la mayor valoración de los nacionales también podría explicarse por el hecho de que la región de Cuenca es conocida por trabajar y elaborar diferentes materiales y productos (barro, joyería, textiles, el propio sombrero de paja toquilla) y este hecho sería más valorado por los turistas ecuatorianos que por los extranjeros por la misma razón que la limpieza o la conservación del entorno. Finalmente, el servicio de taxis y las actividades culturales arrojan unos resultados más variados y difíciles de interpretar. El resultado es una diferencia de 0,23 puntos entre la mejor valoración (la del turista ecuatoriano) y la peor (la del turista europeo), confirmando una cuestión mencionada en la revisión de la literatura: la mayor distancia cultural, genera una peor valoración del destino (Lee et al., 2004).

En relación al número de visitas previas, como puede verse en la tabla 5, la cantidad de veces que se había viajado a la ciudad de Cuenca pareció influir también en la valoración de algunos elementos vinculados a la experiencia turística. En este sentido, para la calificación de la señalización, servicio de taxis, actividad en torno al sombrero de paja toquilla en el Sigsig y, especialmente, comercio de productos artesanos, el número de visitas influiría positivamente en su calificación, siendo elementos que tenderían a valorarse de forma diferenciada con el regreso a Cuenca. Por el contrario, el precio del viaje hasta Cuenca se iría valorando de manera más negativa con la repetición de la visita. En general, el promedio de estos valores tendería a incrementarse conforme al número de visitas, existiendo una diferencia global de 0,14 puntos.

Por último, el conocimiento previo sobre el producto turístico también tendría una diferenciación en la valoración del mismo. De esta forma, como puede verse en la tabla 6 , en todos los valores en los que las diferencias son significativas, la existencia de un conocimiento previo, bien del sombrero de paja toquilla como Patrimonio Inmaterial de la Humanidad, bien como elemento vinculado a la ciudad de Cuenca y su entorno, presentaban una valoración superior a cuando no existía este conocimiento, remarcando la importancia de tratar de llegar en la promoción del destino hasta el visitante en su lugar de origen. 
Tabla 5

VALORACIÓN MEDIA DE LOS ELEMENTOS VINCULADOS A LA SATISFACCIÓN SEGÚN VISITAS PREVIAS

\begin{tabular}{|l|r|r|r|}
\hline Visitas a Cuenca & Nunca & $\begin{array}{c}\text { De 1 a 3 } \\
\text { veces }\end{array}$ & \multicolumn{1}{c|}{$\begin{array}{c}\text { Más de 3 } \\
\text { veces }\end{array}$} \\
\hline Precio del viaje & 3,60 & 3,53 & 3,41 \\
\hline Museos relacionados con el sombrero de paja toquilla & 3,30 & 3,26 & 3,26 \\
\hline Fábricas de sombreros de paja toquilla de Cuenca & 3,23 & 3,20 & 3,24 \\
\hline Cantón de Sigsig y la elaboración del sombrero & 2,49 & 2,73 & 2,66 \\
\hline Hospitalidad/Trato & 4,21 & 4,28 & 4,21 \\
\hline Conservación del entorno & 4,03 & 4,16 & 4,13 \\
\hline Puntos de información y atención al visitante & 3,67 & 3,76 & 3,66 \\
\hline Señalización & 3,50 & 3,62 & 3,72 \\
\hline Alojamiento & 4,14 & 4,11 & 4,03 \\
\hline Restaurantes & 4,07 & 4,18 & 4,14 \\
\hline Seguridad ciudadana & 4,07 & 4,04 & 4,06 \\
\hline Limpieza & 4,14 & 4,16 & 4,16 \\
\hline Conexión a Internet & 3,66 & 3,71 & 3,71 \\
\hline Servicio de Taxis & 3,59 & 3,83 & 3,78 \\
\hline Actividades culturales y espectáculos & 3,61 & 3,82 & 3,84 \\
\hline Zona comercial de productos artesanos & 3,74 & 3,95 & 4,05 \\
\hline Satisfacción fabricación sombrero paja toquilla & 3,87 & 3,90 & 3,99 \\
\hline Opinión Cuenca como Ciudad PCH & 4,44 & 4,51 & 4,52 \\
\hline Satisfacción general viaje & $\mathbf{2 , 9 3}$ & $\mathbf{3 , 0 7}$ & $\mathbf{3 , 0 6}$ \\
\hline PROMEDIO VALORES QUE ARROJAN DIFERENCIAS & & 4,44 & 4,47 \\
\hline
\end{tabular}

Elaboración propia.

Así, la diferencia entre la valoración con la existencia de un conocimiento previo y sin él sería la más amplia de las encontradas hasta ahora, con una distancia de 0,29 puntos para el caso del sombrero de paja toquilla como patrimonio inmaterial y de 0,23 para el conocimiento de que éste es un producto vinculado a Cuenca y su entorno. De esta forma, puede asegurarse que la influencia del conocimiento previo sobre el espacio a visitarse puede influir positivamente en la valoración de algunos de sus elementos, como es el caso de los museos, fábricas y espacios productivos vinculados al mismo, del conocimiento y satisfacción en relación a este producto o de cuestiones vinculadas a las artesanías y las actividades culturales, además de en relación a otros servicios más generales. 


\section{Tabla 6 \\ VALORACIÓN MEDIA DE LOS ELEMENTOS VINCULADOS A LA SATISFACCIÓN SEGÚN CONOCIMIENTO PREVIO}

\begin{tabular}{|l|r|r|r|r|}
\hline \multirow{2}{*}{ Ćtem analizado } & \multicolumn{2}{l|}{$\begin{array}{l}\text { Conocimiento } \\
\text { SPT PIH }\end{array}$} & \multicolumn{2}{c|}{$\begin{array}{c}\text { Conocimiento } \\
\text { CUE SPT }\end{array}$} \\
\cline { 2 - 5 } & \multicolumn{1}{|c|}{ Sí } & \multicolumn{1}{c|}{ No } & \multicolumn{1}{c|}{ Si } & \multicolumn{1}{c|}{ No } \\
\hline Precio del viaje & 3,55 & 3,54 & 3,54 & 3,55 \\
\hline Museos relacionados con el sombrero de paja toquilla & 3,42 & 3,14 & 3,44 & 3,16 \\
\hline Fábricas de sombreros de paja toquilla de Cuenca & 3,42 & 3,03 & 3,46 & 3,07 \\
\hline Cantón de Sigsig y la elaboración del sombrero & 2,83 & 2,39 & 2,81 & 2,47 \\
\hline Hospitalidad/Trato & 4,26 & 4,20 & 4,24 & 4,24 \\
\hline Conservación del entorno & 4,13 & 4,05 & 4,14 & 4,07 \\
\hline Puntos de información y atención al visitante & 3,67 & 3,71 & 3,70 & 3,69 \\
\hline Señalización & 3,62 & 3,55 & 3,59 & 3,59 \\
\hline Alojamiento & 4,09 & 4,13 & 4,09 & 4,13 \\
\hline Restaurantes & 4,14 & 4,09 & 4,12 & 4,11 \\
\hline Seguridad ciudadana & 4,07 & 4,04 & 4,05 & 4,06 \\
\hline Limpieza & 4,20 & 4,10 & 4,21 & 4,10 \\
\hline Conexión a Internet & 3,77 & 3,60 & 3,81 & 3,60 \\
\hline Servicio de Taxis & 3,81 & 3,60 & 3,80 & 3,63 \\
\hline Actividades culturales y espectáculos & 3,81 & 3,66 & 3,84 & 3,66 \\
\hline Zona comercial de productos artesanos & 3,96 & 3,80 & 3,98 & 3,80 \\
\hline Satisfacción fabricación sombrero paja toquilla & 3,96 & 3,85 & 4,02 & 3,83 \\
\hline Opinión Cuenca como Ciudad PCH & 4,52 & 4,43 & 4,47 & 4,48 \\
\hline Satisfacción general viaje & 4,46 & 4,33 & 4,39 & 4,40 \\
\hline PROMEDIO VALORES QUE ARROJAN DIFERENCIAS & $\mathbf{3 , 5 9}$ & $\mathbf{3 , 3 0}$ & $\mathbf{3 , 7 1}$ & $\mathbf{3 , 4 8}$ \\
\hline
\end{tabular}

Elaboración propia.

\section{CONCLUSIONES}

Los destinos culturales se han consolidado como espacios atractivos para ciertos tipos de turistas que buscan alternativas a las vacaciones de "sol y playa", ofreciendo monumentos, gastronomía, fiestas, folklore y otras manifestaciones ligadas a la identidad de un territorio. Pese a que la mayoría de los trabajos sobre turismo cultural se han vinculado a espacios patrimoniales, existe un creciente interés en interpretar este fenómeno en relación con el llamado "patrimonio cultural inmaterial" del que el sombrero de paja toquilla forma parte. Dentro de estos estudios, los factores que pueden influenciar en la satisfacción del turista, desde elementos "naturales" como la edad o el sexo hasta otros "artificiales" como el nivel de ingresos y la profesión, se han convertido en un campo prometedor pero aún poco estudiado. 
En el caso analizado se han identificado elementos socioeconómicos, sociodemográficos y de conocimiento previo. En relación al primer objetivo de la investigación, los resultados han indicado un peso más importante de los últimos, especialmente del número de visitas anteriores y, sobre todo del conocimiento y cercanía hacia el producto turístico. Como señalaban Oom et al., 2011 y Turner et al., 2002, la repetición del viaje y la mayor "cercanía" que éste produce hacia el destino, contribuiría a mejorar la satisfacción del turista. Junto con ellos, el origen parece complementar la diferente valoración de la mayoría de elementos analizados, pudiendo explicar también buena parte de los mismos, como señalan también Oom et al. (2011) quienes indican la importancia de las visitas previas a la hora de valorar un destino turístico, pero añaden nuevas cuestiones a consideración, como la importancia de enfocar la publicidad de un destino y sus atractivos hacia el conocimiento del producto, dado que es también un factor importante en la satisfacción. Por el contrario, otros rasgos socioeconómicos y sociodemográficos, como la edad, el género, el nivel de estudios o la categoría socioprofesional, puestos de relieve por otros autores como Wearing y Wearing (1996), McCleary, Weaver y Hsu (2006) o Kim y Morrison (2005) entre otros, en este caso no contribuirían apenas en la explicación del distinto nivel de satisfacción de los turistas. Por lo tanto, en relación al segundo gran objetivo de este estudio, en el caso de la ciudad de Cuenca, debe destacarse la importancia de la motivación o conocimiento previo sobre el sombrero de paja toquilla, junto al número de veces que se ha repetido la visita y el origen del visitante para explicar las diferencias en cuanto a la calificación de algunos componentes fundamentales de la experiencia turística. De esta manera, un conocimiento previo, una mayor cercanía cultural al espacio visitado y la existencia de visitas previas parecen explicar una mejor valoración de varios componentes, tanto específicos al sombrero de paja toquilla como generales.

La importancia de este estudio estriba en que es capaz de identificar cuáles son algunos de los rasgos que se vinculan con las mayores y menores diferencias a la hora de valorar un destino relacionado con el patrimonio cultural inmaterial. Su principal limitación, por ello mismo, se encuentra en que requeriría de una continuación en busca de qué factores explicarían las diferencias en esos ítems para los cuales aún no se ha encontrado esto, como, por ejemplo, el alojamiento y los restaurantes. Los turistas han demostrado ser sujetos complejos, en los que interactúan diferentes factores en paralelo, que incluso se superponen, para explicar sus diferencias, lo que hace difícil, en cierto modo, obtener una imagen homogénea o "congelada" de los mismos. Por ello, la realización de nuevos trabajos en este tema y su comparación con otros casos de estudio similares pueden resultar de gran importancia para dar explicación a estas cuestiones.

\section{REFERENCIAS}

AGUILAR, M.L. (2011): Tejiendo la vida las artesanías de paja toquilla en el Ecuador. Centro Interamericano de artesanías y artes populares CIDAP, Cuenca, $210 \mathrm{pp}$.

ALEGRE J. y GARAU, J. (2010): «Tourist satisfaction and dissatisfaction», Annals of Tourism Research, vol. 37, $\mathrm{n}^{\circ} 1$, pp. 52-73.

ALEGRE, J. y CLADERA, M. (2009): «Analysing the effect of satisfaction and previous visits on tourist intentions to return», European Journal of Marketing, vol. 43, pp. 670-685. 
BILLE, M. (2012): «Assembling heritage: investigating the UNESCO proclamation of Bedouin intangible heritage in Jordan», International Journal of Heritage Studies, $\mathrm{n}^{\circ}$ 18, pp. 107-123.

BREATHNACH P, et al. (1994): Gender in Irish tourism employment. In Tourism: A Gender Analysis, Kinniard V, Hall D (eds). Wiley: Nueva York; pp. 52-73.

CHEN, C-F. y CHEN, F-S. (2010): «Experience quality, perceived value, satisfaction and behavioral intentions for heritage tourists», Tourism Management, vol. 31, $\mathrm{n}^{\circ} 4$, pp. 29-35.

CORDENTE, M. et al. (2011): «Medición de motivaciones del turismo cultural en ciudades patrimonio de la humanidad», Revista de Análisis Turístico, $\mathrm{n}^{\circ} 12$, pp. 80-85.

DE ROJAS, C. y CAMARERO, C. (2008): «Visitors' experience, mood and satisfaction in a heritage context: Evidence from an interpretation center», Tourism Management, vol. $29, \mathrm{n}^{\circ} 3, \mathrm{pp} .525-537$.

DRASOJEVIC, B. y DJORDJEVIC, B. (2017): «Intangible cultural heritage as tourism. Resource of Serbia», Sociology and Anthropology, vol. 5, n 6, pp. 442-449.

FORMICA, S. y UYSAL, M. (1998): «Market Segmentation of an International Cultural Historical Event in Italy», Journal of Travel Research, vol. 36, n 4, pp. 16-24.

GÓMEZ SCHETTINI, M., ALMIRÓN, A. y GONZÁLEZ BRACCO, M. (2011): «La cultura como recurso turístico de las ciudades, el caso de la patrimonialización del tango en Buenos Aires, Argentina», Estudios y Perspectivas en Turismo, n² 20, pp. 1027-1046.

GONZÁLEZ SANTA-CRUZ, F. y LÓPEZ-GUZMÁN, T. (2017): «Culture, tourism and World HeritageSites», Tourism Management Perspectives, $\mathrm{n}^{\circ}$ 24, pp. 111-116.

HUH, UYSAL y MCCLEARY. (2006): «Cultural/Heritage Destinations: Tourist Satisfaction and Market Segmentation», Journal of Hospitality \& Leisure Marketing, vol.14, ${ }^{\circ} 3$, pp. 81-99.

KEE, T., WAN, D. y HO, A. (2007): «Tourists' satisfaction, recommendation and revisiting Singapore», Tourism Management, vol. 28, n 4, pp. 965-975.

KERSTETTER, D.L., CONFER, J.J. y GRAEFER, A.R. (2001): «An Exploration of the Specialization concept within the context of Heritage Tourism», Journal of Travel Research, vol. 39, n 3, pp. 267-274.

KIM, N. y CHALIP L. (2004): «Why travel to the FIFA World Cup? Effects of motive, background, interest and constraints», Tourism Management, ${ }^{\circ}$ 25, pp. 695-707.

KLAUS WEIERMAIR (2000): «Tourists> perceptions towards and satisfaction with service quality in the cross-cultural service encounter: implications for hospitality and tourism management», Managing Service Quality, vol. 10, n 6, pp. 397-409.

KOZAK, M. y RIMMINGTON, M. (2000): «Tourist Satisfaction with Mallorca, Spain, as an Off-Season Holiday Destination», Journal of Travel Research, $\mathrm{n}^{\circ}$ 38, pp. 260-269.

LEEWORTHY V. R., et al. (2001): «Correcting response bias in tourist spending surveys». Annals of Tourism Research, vol.28, ${ }^{\circ} 1$, pp. 83-97.

LÓPEZ-GUZMÁN, T., et al. (2017): «El patrimonio inmaterial de la humanidad como herramienta de promoción de un destino turístico», Estudios y Perspectivas en Turismo, vol. $26, \mathrm{n}^{\circ} 3$, pp. 568-584.

LÓPEZ-GUZMÁN, T., TORRES-NARANJO, M., PÉREZ-GÁLVEZ, J. y CARVACHEFRANCO, W. (2017): «Segmentation and motivation of foreign tourists in World 
Heritage sistes. A case study, Quito (Ecuador) », Current Issues in Tourism, publicado online: http://www.tandfonline.com/doi/full/10.1080/13683500.2017.1344625 MCCLEARY, K., WEAVER, P. y HSU, C. (2007): «The Relationship Between International Leisure Travelers' Origin Country and Product Satisfaction, Value, Service Quality, and Intent to Return», Journal of Travel \& Tourism Marketing, vol. 21, n 2-3, pp. 117-130. MCKERCHER, B. y DU CROS, H. (2003) : «Testing a cultural tourism typology». International Journal of Tourism Research, vol. 5, pp. 45-58.

NGUYEN, T.H.H., y CHEUNG, C. (2014): «The classification of heritage tourists: a case of Hue City Vietnam», Journal of Heritage Tourism, vol. 9, pp. 35-50.

OOM, P. et al. (2011): «Can welcoming residents increase tourist satisfaction?», Anatolia: An International Journal of Tourism and Hospitality Research, vol. 22, pp. 260-277.

PRADA-TRIGO, J. (2017): «Tourism, territory and cuisine: food consideration and perceptions regarding origin and social changes: the case of Guinea pig», Journal of Tourism and Cultural Change, DOI: 10.1080/14766825.2017.1344678.

PRADA-TRIGO, J., et al. (2015): «De los estudios económicos en turismo a la perspectiva cultural: algunas evidencias metodológicas a partir del caso de estudio de Cuenca (Ecuador)», Anais Brasilero de Estudos Turísticos, vol. 5, n 1, pp. 22-31.

SANZ-IBÁÑEZ, C. y ANTÓN, S. (2014): «The evolution of destinations: towards an evolutionary and relational economic geography approach», Tourism Geographies, vol. 16, pp. 563-579.

SCHOFIELD, P. y THOMPSON, K. (2007): «Behavioural Intention: The 2005 Naadam Festival, Ulaanbaatar», International Journal of Tourism Research, vol. 9, n ${ }^{5}$, pp. 329-344.

TELFER, D. y SHARPLEY, R. (2008): Tourism and Development in the Developing World. Londres: Routledge. 263 pp.

TURNER, L., REISINGER, Y. y MCQUILKEN, L. (2002): «How Cultural Differences Cause Dimensions of Tourism Satisfaction», Journal of Travel \& Tourism Marketing, vol. 11, pp. 79-101.

UNWTO. (2012): Tourism and intangible cultural heritage. Madrid: UNWTO. 130 pp.

VIDAL GONZÁLEZ, M. (2008): «Intangible heritage tourism and identity», Tourism Management, vol. 29, pp. 807-810.

VILLA-MEJÍA, M.C., et al. (2016): «Diseño de productos turísticos culturales a partir del patrimonio inmaterial», Retos Turísticos, vol. 15, pp. 1-16.

WEARING B, WEARING S. (1996): «Refocusing the tourist experience: the 'flaneur' and the 'choraster», Leisure Studies, vol.15, pp. 229-244.

YOON, Y. y UYSAL, M. (2005): «An examination of the effects of motivation and satisfaction on destination loyalty: a structural model», Tourism Management, vol. 26, $\mathrm{n}^{\circ} 1$, pp. 45-56.

YUKSEL, A., YUKSEL F. y BILIM, Y. (2010): «Destination attachment: Effects on customer satisfaction and cognitive, affective and conative loyalty», Tourism Management, vol. 31, pp. 274-284.

ZABKAR, V., MAKOVEC, M. y DMITROVIC, T. (2010): «Modelling perceived quality, visitor satisfaction and behavioural intentions at the destination level», Tourism Management, vol. 31, $\mathrm{n}^{\circ} 4$, pp. 537-546. 
